\title{
EFEITO DE ALTAS TEMPERATURAS NA GERMINAÇÃO DE SEMENTES DE Plathymenia reticulata Benth. E Dalbergia miscolobium Benth. ${ }^{1}$
}

Daniel de Alencastro Bouchardet ${ }^{2}$, Isadora Matos Ribeiro ${ }^{2}$, Nayara de Almeida de Sousa ${ }^{2}$, Stefano Salvo Aires $^{2}$ e Heloisa Sinatora Miranda ${ }^{2}$

\begin{abstract}
RESUMO - O fogo é comum no Cerrado e, geralmente, ocorre durante a seca, quando várias espécies frutificam ou dispersam as sementes. Entretanto, pouco se sabe sobre os efeitos do fogo na germinação das espécies do Cerrado. Este trabalho investigou o efeito da exposição a altas temperaturas na germinação de sementes de Dalbergia miscolobium Benth. e Plathymenia reticulata Benth., comuns no Cerrado e com uso potencial para recuperação de áreas degradadas e na recomposição de áreas de preservação permanente. As sementes foram coletadas em agosto de 2011, pesadas, separadas em três classes de tamanho e expostas a três temperaturas: $80^{\circ} \mathrm{C}, 100{ }^{\circ} \mathrm{C}$ e $150{ }^{\circ} \mathrm{C}$ por 2 e 5 minutos. Após os tratamentos, as sementes foram colocadas para germinar. A exposição a altas temperaturas afetou, de forma diferenciada, a germinação das espécies. Independentemente da classe de tamanho, a exposição das sementes até $100{ }^{\circ} \mathrm{C}$, por $2 \mathrm{e}$ 5 minutos, não alterou a germinação de D. miscolobium (91\%). Entretanto, apenas as sementes maiores germinaram após a exposição a $150^{\circ} \mathrm{C}$, porém com redução significativa na germinação $(53 \%$; $<0,01)$. Para $P$. reticulata, a exposição a $80^{\circ} \mathrm{C}$ por 2 e 5 minutos e a $100^{\circ} \mathrm{C}$ por 2 minutos não alterou a germinação $(71 \%)$, e nenhuma semente germinou após a exposição a $150^{\circ} \mathrm{C}$. A baixa resistência das sementes às altas temperaturas pode afetar significativamente o recrutamento de novos indivíduos após a ocorrência de queimadas no Cerrado.
\end{abstract}

Palavras-chave: Dalbergia miscolobium; Plathymenia reticulata; Altas temperaturas.

\section{EFFECT OF HIGH TEMPERATURES ON GERMINATION OF Plathymenia reticulata Benth. AND Dalbergia miscolobium Benth.}

\begin{abstract}
Fire is common in the Cerrado occurring during the dry season when many species fruit or disperse the seeds. However, little is known about the fire effect on seed germination. This study aimed to investigate the effect of high temperatures on seed germination of Dalbergia miscolobium Benth. and Plathymenia reticulata Benth., common species of the Cerrado and with potential use in recovery of degraded areas and restoration of permanent preservation areas. Seeds were collected in August 2011, weighed, separated into three size classes and exposed to three temperatures: $80^{\circ} \mathrm{C}, 100^{\circ} \mathrm{C}$ and $150{ }^{\circ} \mathrm{C}$ for 2 and 5 minutes. After the treatments, the seeds were germinated. Exposure to high temperatures had different effects in germination of species. Regardless of size class, the seed exposure up to $100{ }^{\circ} \mathrm{C}$ for 2 and 5 minutes did not alter germination of D. miscolobium seeds (91\%). However, after exposure to $150^{\circ} \mathrm{C}$ only the seeds with higher mass germinated, but with a significant reduction in germination (53\%; $p<0.01$ ). Exposure to $80^{\circ} \mathrm{C}$ for 2 and 5 minutes and to $100{ }^{\circ} \mathrm{C}$ for 2 minutes did not affect $\boldsymbol{P}$. reticulata germination (71\%). No seed germinated after exposure to $150^{\circ} \mathrm{C}$. The low resistance of the seeds to high temperatures can significantly affect the recruitment of new individuals after the occurrence of Cerrado fires.
\end{abstract}

Keywords: Dalbergia miscolobium; Plathymenia reticulata; High temperatures.

\footnotetext{
${ }^{1}$ Recebido em 05.12.2012 aceito para publicação em 15.05.2015.

${ }^{2}$ Universidade de Brasília, UNB, Instituto de Ciências Biológicas, Departamento de Ecologia, Asa Norte, Brasília, DF - Brasil. E-mail:<dbouchardet@gmail.com>,<isadora_ribeiro_8@hotmail.com>,<naya.sousa@gmail.com>,<stefanoaires@gmail.com> e $<$ hmiranda@unb.br>.
} 


\section{INTRODUÇ̃̃O}

O fogo é comum no Cerrado, podendo ser natural ou antrópico (FRANÇA et al., 2007) e está presente nesse bioma há mais de 32.000 A.P. (FERRAZVICENTINI, 1999). Características como alta suberização do tronco e dos galhos, que permitem certo grau de isolamento térmico (COUTINHO, 1990; GUEDES, 1993), a capacidade de rebrotar (COUTINHO, 1990) e a capacidade dos frutos de isolarem suas sementes do calor (CIRNE; MIRANDA, 2008) indicam a adaptação da vegetação do Cerrado ao fogo. Embora existam diversos trabalhos sobre a relação entre o fogo e a vegetação do Cerrado (SATO et al., 2010; MUNHOZ; AMARAL, 2010), o efeito das altas temperaturas na germinação de sementes é pouco estudado, e os trabalhos apresentam resultados contrastantes (ZAIDAN; CARREIRA, 2008; ANDRADE; MIRANDA, 2010). Para gramíneas, Overbeck et al. (2006) apresentaram dois padrões diferentes: Andropogon lateralis e Shizachyrium microstachium não têm a germinação alterada quando as sementes são expostas até $110^{\circ} \mathrm{C}$ por $2 \mathrm{~min}$, enquanto Leptochoryphium lanatum e Elionurus muticus apresentam redução na germinação a partir da exposição a $70^{\circ} \mathrm{C}$, por $2 \mathrm{~min}$. Considerando outras espécies do estrato herbáceo-arbustivo, sementes de Sygonanthus nitens não apresentam alteração na germinação quando exposta à temperatura de até $200^{\circ} \mathrm{C}$ por $1 \mathrm{~min}$, entretanto a exposição a $200^{\circ} \mathrm{C}$ por 3 e 5 min reduz significativamente a germinação (FICHINO et al., 2012), e sementes de Heteropterys pteropetala mostram decréscimo na germinação a partir da exposição a $100^{\circ} \mathrm{C}$ por $5 \mathrm{~min}$ (SCHMIDT et al., 2005). Para Kilmeyera coriacea, espécie do estrato arbóreo, Cirne e Miranda (2008) mostraram que a passagem do fogo favorece a abertura dos frutos e não altera a germinação. Ainda, Andrade et al. (1997) reportaram aumento na germinação das sementes de Bowdichia virgilioides com o incremento da temperatura. De forma geral, esses resultados sugerem que tanto a temperatura quanto a duração do pulso de calor estão relacionadas com a alteração da germinação, embora essas alterações também possam estar relacionadas à liberação de etileno ou de amônia durante a queimada e ao ressecamento ou quebra do tegumento, facilitando a embebição e aumentando a germianação ou causando a morte do embrião, com redução no recrutamento pósfogo (GASHAW; MICHELSON, 2002).

Gashaw e Michelson (2002) postularam que sementes maiores demoram mais tempo para atingir o equilíbrio com a temperatura ambiente, portanto o embrião ficará menos tempo exposto a altas temperaturas. De acordo com Lahoreau et al. (2006), sementes menores teriam menor chance de sucesso no estabelecimento por apresentarem menor conteúdo de reserva. Popinigis (1985) afirmou que, para uma espécie, as sementes com menor tamanho e, ou, menor densidade possuem menor qualidade fisiológica, como observado por Sassaki e Felippe (1999) para Dalbergia miscolobuim.

Este trabalho teve como objetivo caracterizar a taxa de germinação de sementes de diferentes tamanhos de Dalbergia miscolobium Benth e Plathymenia reticulata Benth quando expostas a temperaturas de $80{ }^{\circ} \mathrm{C}, 100{ }^{\circ} \mathrm{C}$ e $150{ }^{\circ} \mathrm{C}$ por 2 e 5 min. Essa situação é comum durante queimadas de Cerrado (MIRANDA et al., 2009), considerando-se que essas espécies são pioneiras no seu uso potencial para recuperação de áreas degradadas e de recomposição de áreas de preservação permanente (LORENZI, 2008).

\section{MATERIAL E MÉTODOS}

Dalbergia miscolobium (Fabaceae), popularmente chamada de jacarandá-do-cerrado, está presente naturalmente em Cerrado sentido restrito e Cerradão distrófico. É uma espécie pioneira com altura que varia entre 8 e $16 \mathrm{~m}$ e diâmetro entre 30 e $50 \mathrm{~cm}$. Sua madeira é moderadamente pesada, com densidade de $0,81 \mathrm{~g} /$ $\mathrm{cm}^{3}$, sendo apropriada para fabricação de pequenos objetos, acabamento interno e móveis. É uma árvore decídua, com folhação acontecendo nos meses de julho a setembro. A floração ocorre de novembro a maio e a frutificação, de maio a julho (SILVA JÚNIOR, 2005; LORENZI, 2008). Produz grande quantidade de frutos, e as sementes são dispersas pelo vento. De acordo com Sassaki e Felippe (1999) e Ribeiro et al. (2007), apresenta baixa porcentagem de sementes predadas ( $0 \%$ a $13 \%$ ) e abortadas ( $13 \%$ a $43 \%)$, e a germinação é alta (80\% a 99\%). Para características das sementes, ver Sassaki e Felippe (1999).

Plathymenia reticulata (Fabaceae), conhecida como Vinhático, ocorre em formações abertas de Cerrado e em áreas de transição para florestas semidecíduas. A altura dos indivíduos varia de 6 a 12 m e o diâmetro do tronco, de 30 a $50 \mathrm{~cm}$. Possui uso potencial para recuperar áreas degradadas, pois é uma espécie pioneira. Sua madeira é de densidade leve $\left(0,55 \mathrm{~g} / \mathrm{cm}^{3}\right)$, fácil de ser manuseada em carpintaria e possui alta resistência natural a organismos xilófagos. Floresce de setembro 
até meados de novembro, e os frutos amadurecem nos meses de agosto e setembro do ano seguinte, enquanto a copa está totalmente sem folhas (LACERDA et al., 2004; LORENZI, 2008). Ribeiro et al. (2007) reportaram que os frutos apresentam alta porcentagem de sementes abortadas (10\% a 64\%) e baixa porcentagem de sementes predadas (13\% a 19\%). A taxa de germinação varia entre 22\% e 90\% (BRAGA et al., 2007; RIBEIRO et al., 2007). Para características das sementes, ver Lopes et al. (2010).

Frutos maduros de D. miscolobium e P. reticulata foram coletados, em agosto de 2011 , de 10 matrizes em uma área de Cerrado próxima a Brasília, Distrito Federal. A distância entre as matrizes foi de, no mínimo, $50 \mathrm{~m}$ para assegurar variabilidade genética, conforme sugerido por Pinã-Rodrigues et al. (2004). Todos os frutos foram coletados, ao acaso, na copa das árvores como em Lopes et al. (2010). Para evitar a redução na taxa de germinação, as sementes foram beneficiadas logo após a coleta (BRAGA et al., 2007). As sementes que apresentavam sinais de predação e, ou, de má formação foram descartadas.

Para testar a influência do tamanho das sementes na germinação, as sementes selecionadas foram pesadas e, de acordo com a metodologia descrita por Sassaki e Felippe (1999), divididas em três classes: pequenas, médias e grandes. Para D. miscolobium, as sementes pequenas possuíam massa entre 20,0 e $50,0 \mathrm{mg}$, as médias entre 60,0 e 80,0 mg e as grandes acima de 90,0 mg. Para $P$. reticulata, a massa das sementes pequenas variou entre 13,9 e $32,5 \mathrm{mg}$, as médias entre 35,0 e 45,0 $\mathrm{mg}$ e as grandes, acima de 47,5 $\mathrm{mg}$. A descontinuidade na divisão das massas é proposta por Sassaki e Felippe (1999), para evitar a sobreposição de classes.

Para a análise do efeito da temperatura e do tempo de exposição na germinação, as sementes de cada classe de tamanho foram submetidas a fluxo de ar quente em estufa ajustada a três temperaturas: $80{ }^{\circ} \mathrm{C}, 100{ }^{\circ} \mathrm{C}$ e $150{ }^{\circ} \mathrm{C}$, por 2 e $5 \mathrm{~min}$. Para $P$. reticulata, foram utilizadas três repetições com 10 sementes para cada combinação de classe de tamanho, temperatura e tempo de exposição. Para D. miscolobium, quatro repetições com 30 sementes de cada combinação de classe de tamanho, temperatura e tempo de exposição foram utilizadas. O número de sementes por repetição foi estabelecido em função da classe com menor frequência, como sugerido por Bell et al. (1995). Para as duas espécies, a disponibilidade de sementes pequenas limitou o número de repetições.
De acordo com Lopes et al. (2010), sementes pequenas de $P$. reticulata geralmente estão localizadas próximas às extremidades ou nas reentrâncias dos frutos e são mais sujeitas ao aborto. Já em D. miscolobium sementes menores são encontradas em frutos com duas sementes, que são raros, em que, geralmente, a semente basal é menor do que a apical (SASSAKI; FELIPPE, 1999). Para cada espécie e classe de tamanho, sementes não expostas ao fluxo de calor formaram o grupo-controle, com o mesmo número de sementes e de repetições utilizado nos tratamentos.

Após os tratamentos, as sementes foram colocadas para germinar em placas de Petri forradas com papelfiltro. As placas foram dispostas, ao acaso, em prateleiras sob luz branca, com fotoperíodo de $12 \mathrm{~h}$ e em temperatura ambiente. Diariamente, por 30 dias ou até que não houvesse mais sementes viáveis, foi feita a contagem das sementes germinadas e, se necessário, o papelfiltro era umedecido. O critério utilizado para contabilizar a germinação foi a emergência e curvatura geotrópica da radícula (LABOURIAU, 1983). Após a contagem, as sementes germinadas foram retiradas das placas. Não foi realizado o teste de viabilidade com tetrazólio para as sementes que não germinaram por apresentar sinais de apodrecimento, como necrose e enegrecimento do tecido. Para calcular o tempo médio de germinação, foi utilizada a fórmula descrita por Santana e Ranal (2004).

Os dados de germinação foram transformados em $\operatorname{arcosseno}(\sqrt{ }(\% / 100))$ e submetidos à análise de variância (ANOVA fator único com Tukey a posteriori), sendo adotado o nível de significância de 5\%. Três fatores foram utilizados para avaliar o efeito da temperatura, do tempo de exposição e do tamanho das sementes na germinação. Os valores de tempo médio de germinação foram testados quanto à normalidade e homogeneidade e, devido à alta variabilidade, foram analisados por meio do teste de Kruskall-Wallis $(\alpha=0,05)$ com teste de Dunn a posteriori.

\section{RESULTADOS}

As sementes de Dalbergia miscolobium apresentaram germinação rápida com mais de 50\% das sementes germinando até o quinto dia. A germinação encerrou-se após 14 dias, com 91\% das sementes germinadas. Sementes pequenas apresentaram menor tempo médio de germinação ( 4 dias, $\mathrm{H}=5,3333$; $\mathrm{p}=$ $0,0209)$ que as sementes médias e grandes ( 5 dias). 
Para as sementes expostas ao fluxo de ar quente, o tamanho da semente, a temperatura e o tempo de exposição não influenciaram o tempo médio de germinação. Platimenia reticulata apresentou germinação mais lenta que a primeira espécie, com 50\% da germinação sendo registrada no décimo dia e encerrando no vigésimo nono dia, com $70 \%$ de sementes germinadas. O tempo de exposição e a temperatura não influenciaram o tempo médio de germinação, exceto quando expostas a $100{ }^{\circ} \mathrm{C}$ por $5 \mathrm{~min}$, quando foi registrado atraso na germinação das sementes grandes ( 17 dias, $\mathrm{H}=3,8571 ; \mathrm{p}=0,0495$ ) em relação às médias (10 dias) e pequenas (7 dias).

Sementes de Dalbergia miscolobium que não foram expostas ao fluxo de calor apresentaram germinação de $91 \%$, não tendo sido registrada diferença significativa entre as classes de tamanho (Figura 1). As sementes pequenas e médias não apresentaram redução na germinação quando aquecidas a temperaturas de até $100{ }^{\circ} \mathrm{C}$ por $5 \mathrm{~min}$, porém nenhuma semente germinou após a exposição a $150^{\circ} \mathrm{C}$ por 2 ou 5 min (Tabela 1 ). Para as sementes grandes, houve redução significativa

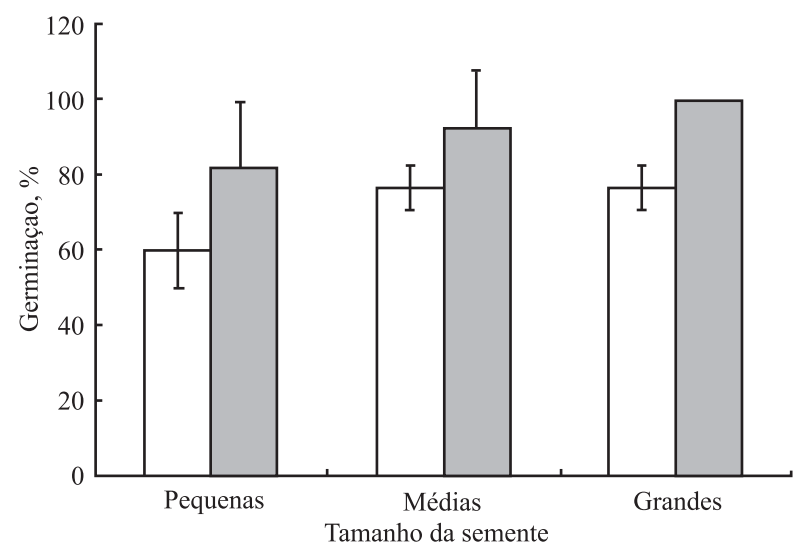

Figura 1 - Germinação de sementes de diferentes tamanhos de Plathymenia reticulata ( $\square$ ) e Dalbergia miscolobium (ロ). P. reticulata: Pequenas $=13,9$ a $32,5 \mathrm{mg}$; Médias $=35,0$ a 45,0 $\mathrm{mg}$; Grandes $>47,5 \mathrm{mg}$. D. miscolobium: Pequenas $=20,0$ a $50,0 \mathrm{mg}$; Médias $=60,0$ a 80,0 mg; Grandes $>$ $90,0 \mathrm{mg}$. Comparações $(\alpha=0,05)$ dentro da mesma espécie.

Figure 1-Germination of seeds of different sizes of Plathymenia reticulata ( $\square$ ) and Dalbergia miscolobium ( $\square$ ). P. reticulata: Small = 13.9 to $32.5 \mathrm{mg}$; Medium $=35.0$ to $45.0 \mathrm{mg}$; Large $>47.5 \mathrm{mg}$. D. miscolobium: Small $=20.0$ to $50.0 \mathrm{mg}$; Medium $=60.0$ to $80.0 \mathrm{mg} ;$ Large $>90.0 \mathrm{mg}$. Comparison in the same species $(\alpha=0.05)$. na germinação $(\mathrm{Q}=5,8961 ; \mathrm{p}<0,01)$ após a exposição a $150{ }^{\circ} \mathrm{C}$ por $5 \mathrm{~min}$. Dos parâmetros considerados (temperatura, tempo de exposição e tamanho das sementes), apenas o tempo de exposição não afetou significativamente a germinação (Tabela 2).

Para Plathymenia reticulata, a taxa de germinação de sementes não expostas ao fluxo de calor foi de $71 \%$, não havendo diferença significativa entres as classes de tamanho (Figura 1). As sementes que foram aquecidas não apresentaram diferença significativa na germinação quando submetidas a $80^{\circ} \mathrm{C}$, independentemente do tempo de exposição, e a $100^{\circ} \mathrm{C}$ por $2 \mathrm{~min}$; entretanto, a exposição das sementes a $100^{\circ} \mathrm{C}$ por 5 min reduziu significativamente a germinação, sendo a redução mais acentuada nas sementes pequenas $(Q=6,7435$; $p<0,01$ - Tabela 1). Nenhuma semente germinou após a exposição a $150{ }^{\circ} \mathrm{C}$. Tanto a temperatura quanto o tempo de exposição e o tamanho das sementes afetaram significativamente a germinação. Entretanto, a interação entre o tempo de exposição e o tamanho das sementes não se mostrou significativa (Tabela 2).

\section{DISCUSSÃO}

Nas sementes que não foram expostas ao fluxo de ar quente, não foi observada diferença significativa na germinação de sementes de diferentes tamanhos (Figura 1). Embora Sassaki e Felippe (1999) tenham registrado as menores taxas de germinação de sementes pequenas de D. miscolobium, essa diferença pode estar associada à variação da massa das sementes, uma vez que as sementes de D. miscolobium utilizadas neste estudo apresentaram massa semelhante àquelas consideradas como pequenas por Sassaki e Felippe (1999). As sementes de $P$. reticulata apresentaram pequena variação na massa (13,9 a 47,5 mg), semelhante ao apresentado por Aguiar et al. (1979) para sementes de Eucalyptus grandis e Eucalyptus urophylla (14 a 59 mg) e que não apresentaram variação na germinação em relação ao tamanho da semente.

A exposição das sementes ao ar quente resultou em redução significativa na germinação, tanto para D. miscolobium quanto para P. reticulata (Tabela 1), o que também foi observado por Schmidt et al. (2005), Overbeck et al. (2006) e Fichino et al. (2012) em várias espécies do Cerrado. Embora tenha sido registrada redução significativa na germinação de sementes de $P$. reticulata a $100^{\circ} \mathrm{C}$ por $5 \mathrm{~min}$, independentemente 
Tabela 1 - Germinação de Plathymenia reticulata e de Dalbergia miscolobium exposta a $80{ }^{\circ} \mathrm{C}, 100{ }^{\circ} \mathrm{C}$ e $150{ }^{\circ} \mathrm{C}$ por 2 e 5 minutos. Letras diferentes indicam diferença significativa $(\alpha=0,05)$ entre os valores. Letras maiúsculas indicam comparação entre tratamentos e as minúsculas, entre tamanhos.

Table 1 - Germination of Plathymenia reticulata and Dalbergia miscolobium after exposure to $80{ }^{\circ} \mathrm{C}, 100{ }^{\circ} \mathrm{C}$ and 150 ${ }^{\circ} \mathrm{C}$ for 2 and 5 minutes. Different letters correspond to significant differences $(\alpha=0.05)$. Capital letters correspond to comparisons among treatments and lower cases comparisons among sizes.

\begin{tabular}{|c|c|c|c|c|c|c|c|}
\hline \multirow{3}{*}{ Tamanhodasemente } & & \multicolumn{6}{|c|}{ Germinação (\%) } \\
\hline & \multirow[t]{2}{*}{ Controle } & \multicolumn{2}{|c|}{$80^{\circ} \mathrm{C}$} & \multicolumn{2}{|c|}{$100^{\circ} \mathrm{C}$} & \multicolumn{2}{|c|}{$150^{\circ} \mathrm{C}$} \\
\hline & & 2 minutos & 5 minutos & 2 minutos & 5 minutos & 2 minutos & 5 minutos \\
\hline \multicolumn{8}{|c|}{ Dalbergia miscolobium } \\
\hline Pequena & $81,7 \mathrm{Aa}$ & $91,6 \mathrm{Aa}$ & $63,3 \mathrm{Aa}$ & $83,3 \mathrm{Aa}$ & $80,8 \mathrm{Aa}$ & 0 & 0 \\
\hline Média & $92,5 \mathrm{Aa}$ & $90,8 \mathrm{Aa}$ & $90,0 \mathrm{Aa}$ & $92,5 \mathrm{Aa}$ & $96,7 \mathrm{Aa}$ & 0 & 0 \\
\hline Grande & $100,0 \mathrm{Aa}$ & $92,5 \mathrm{Aa}$ & $92,5 \mathrm{Aa}$ & $87,5 \mathrm{Aa}$ & $89,7 \mathrm{Aa}$ & $75,0 \mathrm{AB}$ & $53,3 \mathrm{~B}$ \\
\hline \multicolumn{8}{|l|}{ Plathymenia reticulata } \\
\hline Pequena & $60,0 \mathrm{Aa}$ & $60,0 \mathrm{Aa}$ & $53,3 \mathrm{Aa}$ & $53,3 \mathrm{Aa}$ & $6,7 \mathrm{Ba}$ & 0 & 0 \\
\hline Média & $76,7 \mathrm{Aa}$ & $80,0 \mathrm{Aa}$ & $83,3 \mathrm{Aa}$ & $66,7 \mathrm{Ab}$ & $60,0 \mathrm{Ab}$ & 0 & 0 \\
\hline Grande & $76,7 \mathrm{Aa}$ & $66,7 \mathrm{Aa}$ & $83,3 \mathrm{Aa}$ & $93,3 \mathrm{Ab}$ & $30,0 \mathrm{Bab}$ & 0 & 0 \\
\hline
\end{tabular}

Dalbergia miscolobium - Pequena: 20,0 a 50,0 mg: Média: 60,0 a 80,0 mg e Grande $>90,0 \mathrm{mg}$.

Plathymenia reticulata - Pequena: 13,9 a 32,5 mg; Média: 35,0 a 45,0 mg e Grande: $>47,5 \mathrm{mg}$.

Tabela 2 - Fonte de variação: resultados do ANOVA três fatores.

Table 2 - Source of variation. Three-Factor ANOVA result.

\begin{tabular}{lcc}
\hline \multirow{2}{*}{ Fonte de variação } & \multicolumn{2}{c}{ Germinação (\%) } \\
\cline { 2 - 3 } & Dalbergia miscolobium & $* *$ \\
\hline Temperatura $(\mathrm{T})$ & $* *$ & $* *$ \\
Tempo de exposição (t) & NS & $* *$ \\
Tamanho da semente (S) & $* *$ & $* *$ \\
T x t & NS & $*$ \\
T x S & $* *$ & NS \\
t $x$ S & NS
\end{tabular}

$* *<0,01 ; *<0,05$.

do tamanho, para D. miscolobium apenas sementes grandes germinaram após a exposição a $150{ }^{\circ} \mathrm{C}$, com redução significativa apenas após a exposição a essa temperatura por $5 \mathrm{~min}$. A diferença na capacidade de sementes de diferentes tamanhos tolerarem altas temperaturas tem sido amplamente discutida na literatura (JONES; NIELSON, 1999; HANLEY et al., 2003) e, de acordo com Gashaw e Michesen (2002), sementes maiores levam um tempo maior para atingir o equilíbrio com a temperatura ambiente, de tal forma que seus embriões estarão expostos por um tempo menor a altas temperaturas, como observado por Delgado et al. (2001) em sementes de Cistus ladanifer.

O tempo médio de germinação não apresentou alterações significativas em relação ao tamanho das sementes, tratamentos térmicos e tempo de exposição das sementes de D. miscolobium, sendo, portanto, um parâmetro menos sensível em relação à germinabilidade para essa espécie. Quando expostas à temperatura de $100^{\circ} \mathrm{C}$ por $5 \mathrm{~min}$, sementes pequenas de $P$. reticulata apresentaram redução no tempo médio de germinação, e as sementes grandes aumentaram. Embora a exposição a altas temperaturas acompanhada de tempo de exposição prolongado possa ocasionar desnaturação de proteínas, alteração nas atividades enzimáticas necessárias à germinação e até mesmo danos mais severos nas estruturas celulares, que podem resultar em atraso ou bloqueio germinativo (CARVALHO et al., 2001; BORGHETTI; FERREIRA, 2004), nas sementes pequenas, o baixo número de sementes germinadas pode ter causado um viés no cálculo do tempo médio de germinação. Nesse caso, a média ponderada utilizada pelo cálculo seria alterada, levando

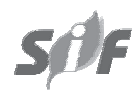

Revista Árvore, Viçosa-MG, v.39, n.4, p.697-705, 2015 
a uma interpretação errônea de germinação acelerada para essa classe de sementes (SANTANA; RANAL, 2004).

Os resultados obtidos de D. miscolobium e $P$. reticulata sugerem que o fogo pode afetar significativamente o recrutamento de novos indivíduos. Durante as queimadas de Cerrado, a temperatura na superfície do solo é da ordem de $280^{\circ} \mathrm{C}$ (CASTRONEVES; MIRANDA, 1996), indicando que as sementes localizadas na superfície do solo que não forem consumidas pelo fogo terão a germinabilidade significativamente reduzida ou serão inviabilizadas. Entretanto, nas sementes ainda nos frutos, o escape ao efeito das altas temperaturas pode ser determinado pela posição dos frutos na copa. Embora as queimadas de Cerrado sejam de superfície (MIRANDA et al., 2009), a altura das chamas varia entre 1,2 m e 2,9 m (CASTRO; KAUFFMANN, 1998), podendo causar danos diretos a frutos localizados nessa faixa de altura (LANDIM; HAY, 1996; RAMOS, 2004; CIRNE; MIRANDA, 2008; PALERMO, 2011) e indiretos àqueles expostos ao fluxo de ar quente, mesmo que o fruto ofereça isolamento térmico para as sementes (JUDD, 1993), como observado por Gleadow e Narayan (2007) para Pittosporum undulatum durante queimadas na Austrália. Para Kielmeyera coriacea, Cirne e Miranda (2008) mostraram que, dependendo da altura na copa em que o fruto se encontra, a temperatura pode atingir valores entre $390^{\circ} \mathrm{C} \mathrm{e} 730^{\circ} \mathrm{C}$, reforçando as observações de Landim e Hay (1996), que relacionaram os danos em frutos de $K$. coriacea à sua posição na copa. $\mathrm{O}$ escape aos efeitos das altas temperaturas durante queimadas também foi reportado em Mimosa clausenii e Qualea parviflora. Ramos (2004) mostrou que, após uma queimada em Cerrado sensu stricto, nenhuma semente de $M$. clausenii localizada até $1 \mathrm{~m}$ de altura germinou e, para sementes de frutos localizados entre $1 \mathrm{~m}$ e $2 \mathrm{~m}$, houve maior redução da germinabilidade (de $97 \%$ para $32 \%$ ) do que para as de frutos localizados acima de $2 \mathrm{~m}$ (de $87 \%$ para $52 \%$ ). Perdas semelhantes foram reportadas para sementes de Q. parviflora: sementes localizadas abaixo de $1 \mathrm{~m}$ não germinaram, e as localizadas acima dessa altura reduziram a germinação de $90 \%$ para menos de $20 \%$ (PALERMO, 2011). Dessa forma, queimadas de Cerrado durante o período de pré-dispersão podem reduzir a germinação das sementes de D. miscolobium e $P$. reticulata e, consequentemente, o recrutamento de novos indivíduos.

Revista Árvore, Viçosa-MG, v.39, n.4, p.697-705, 2015

\section{CONCLUSÃO}

A exposição das sementes a altas temperaturas não estimulou a germinação das sementes das espécies estudadas. Ao contrário, a exposição resultou em efeito negativo na germinação das sementes de $D$. miscolobium e de $P$. reticulata. Independentemente do tamanho, sementes de $P$. reticulata não germinaram quando expostas a temperaturas superiores a $100{ }^{\circ} \mathrm{C}$, e somente as sementes grandes de D. miscolobium germinaram a $150^{\circ} \mathrm{C}$, porém com redução no número de sementes germinadas. Portanto, a germinação das sementes das duas espécies estudadas pode ser significativamente reduzida após a ocorrência de um incêndio, uma vez que, tanto na superfície do solo quanto na zona de chamas, as temperaturas são superiores às utilizadas neste trabalho. Dessa forma, apenas os frutos localizados nos ramos mais altos de indivíduos adultos não serão afetados. Considerando a redução no número de sementes viáveis com possível impacto negativo no recrutamento de novos indivíduos na área, o uso do fogo no manejo de áreas de Cerrado deve levar em consideração não apenas a resistência de indivíduos adultos ao fogo, mas também seus impactos no ciclo reprodutivo.

\section{AGRADECIMENTOS}

Ao Programa ProIC-CNPq-UnB, pela bolsa concedida ao primeiro autor; à CAPES, pela bolsa de doutorado concedida a Stefano Salvo Aires; e às Dras. Sarah C. C. Oliveira e Margarete N. Sato, pelas valiosas sugestões na revisão do manuscrito.

\section{REFERÊNCIAS}

AGUIAR, I.B.; CARVALHO, N.M.; MAIMONIRODELLA, R.C.S.; DAMASCENO, M.C.M. Influência do tamanho sobre a germinação e o vigor de sementes de eucalipto. Revista Brasileira de Sementes, v.1, n.1, p.53-58, 1979.

ANDRADE, A.C.S; LOUREIRO, M.B.; SOUZA, A.D.O.; RAMOS, F.N. Quebra de dormência de sementes de Sucupira preta. Pesquisa

Agropecuária Brasileira, v. 32, n.5, p.465-469, 1997.

ANDRADE, L.A.Z.; MIRANDA, H.S. O fator fogo no banco de sementes. In: MIRANDA, H. S.

(Org.). Efeitos do regime do fogo sobre 
a estrutura de comunidades de cerrado: resultados do Projeto Fogo. Brasília: IBAMA, 2010. p.103-119.

BELL, D.T.; ROCKICH, D.P.; McCHESNEY, C.J.; PLUMMER, J.A. Effects of temperature, light and gibberellic acid on the germination of seeds of 43 species native to Western Australia. Journal of Vegetation Science, v.6, n.6, p.797-806, 1995.

BORGHETTI, F.; FERREIRA, A.G. Interpretação de resultados de germinação. In: FERREIRA, A.G.; BORGHETTI, F. (Ed.). Germinação: do básico ao aplicado. Porto Alegre: Artmed, 2004. p.251-262.

BRAGA, L.L; TOLENTINO, G.S; SANTOS, M.R.; VELOSO, M.D.M.; NUNES, Y.R.F. Germinação de sementes de Plathymenia reticulata Benth. (Fabaceae-Mimosoideae) sob influência do tempo de armazenamento. Revista Brasileira de Biociências, v.5, supl.2, p.258-260, 2007.

CARVALHO, P.G.B.; BORGETTI, F.; BUCKERIDGE, M.S.; MORHY, L.; FERREIRA FILHO, E.X. Temperature dependent germination and endo beta mannanase activity in sesame seeds. Revista Brasileira de Fisiologia Vegetal, v.13, n.2, p.139-148, 2001.

CASTRO, E.A.; KAUFFMANN, J.B. Ecosystem structure in the Brazilian Cerrado: a vegetation gradient of aboveground biomass, root mass and consumption by fire. Journal of Tropical Ecology, v.14, p.263-283, 1998.

CASTRO-NEVES, B.M.; MIRANDA, H.S. Efeitos do fogo no regime térmico do solo de um campo sujo de Cerrado. In: MIRANDA, H.S.; SAITO, C.H.; DIAS, B.F.S. (Org.). Impactos de queimadas em áreas de cerrado e restinga. Brasília: ECL/UnB, 1996. p.20-30.

CIRNE, P.; MIRANDA, H.S. Effects of prescribed fires on the survival and release of seeds of Kielmeyera coriacea (Spr.) Mart. (Clusiaceae) in savannas of Central Brazil. Brazilian Journal Plant Physiology, v.20, n.3, p.197-204, 2008.

COUTINHO, L.M. Fire in the ecology of Brazilian cerrado. In: GOLDAMMER, J.G. (Ed.). Fire in the tropical biota. Ecological studies. Berlin: Springer Verlag, 1990. p.82-105.
DELGADO, J.A.; SERRANO, J.M.; LÓPEZ, F.; ACOSTA, F.J. Heat shock, mass-dependent germination, and seed yield as related components of fitness in Cistus ladanifer. Environmental and Experimental Botany, v.46, n.1, p.11-20, 2001.

FERRAZ-VICENTINI, K.R. História do fogo no Cerrado: uma análise palinológica. 1999. 207f. Tese (Doutorado em Ecologia) Universidade de Brasília, Brasília, 1999.

FICHINO, B.; FIDELIS, A.; SCHMIDT, I.; PIVELLO, V. Efeitos de altas temperaturas na germinação de sementes de capim-dourado (Syngonanthus nitens) (Bong.) Ruhland (Eriocaulaceae): implicações para o manejo. Acta Botanica Brasilica, v.26, n.2, p.512-515, 2012.

FRANÇA, H.; RAMOS-NETO, M.B.; SETZER, A. $O$ fogo no Parque Nacional das Emas. Brasília: Ministério do Meio Ambiente, 2007. 140p. (Série Biodiversidade, 27).

GASHAW, M.; MICHELSEN, A. Influence of heat shock on seed germination of plants from regularly burnt savanna and grasslands in Ethiopia. Plant Ecology, v.159, n.1, p.83-93, 2002.

GLEADOW, R.M.; NARAYAN, I. Temperature thresholds for germination and survival of Pittosporum undulatum: implications for management by fire. Acta Oecologica, v.31, n.2, p.151-157, 2007.

GUEDES, D.M. Resistência das árvores do Cerrado ao fogo: papel da casca como isolante térmico. 1993. 99f. Dissertação (Mestrado em Ecologia) - Universidade de Brasília, Brasília, 1993.

HANLEY, M.E.; UNNA, J.E.; DARVILL, B. Seed size and germination response: a relationship for fire-following plant species exposed to thermal shock. Oecologia, v.134, n.1, p.18-32, 2003.

JONES, T.A.; NIELSON, D.C. Intrapopulation genetic variation for seed dormancy in Indian ricegrass. Journal of Range Management, v.52, n.6, p.646-650, 1999.

JUDD, T.S. Seed survival in small myrtaceous capsules subjected to experimental heating. Oecologia, v.93, n.4, p.576-581, 1993.

Revista Árvore, Viçosa-MG, v.39, n.4, p.697-705, 2015 
LABOURIAU, L.G. A germinação das sementes. Washington: OEA, 1983. 138p.

LACERDA, D.R.; LEMOS FILHO, J.P.; GOULART, M.F.; RIBEIRO, R.A.; LOVATO, M.B. Seeddormancy variation in natural populations of two tropical leguminous tree species: Senna multijuga (Caesalpinoideae) and Plathymenia reticulata (Mimosoideae). Seed Science Research, v.14, p.127-135, 2004.

LAHOREAU, G.; BAROT, S.; GIGNOUX, J.; HOFFMANN, W.A.; SETTERFIELD, S.A.; WILLIANS, P.R. Positive effect of seed size on seedling survival in fire-prone savannas of Australia, Brazil and West Africa. Journal of Tropical Ecology, v.22, n.6, p.719-722, 2006.

LANDIM, M.F.; HAY, J.D. Impacto do fogo sobre alguns aspectos da biologia reprodutiva de Kielmeyera coriacea Mart. Revista Brasileira de Biologia, v.56, n.1, p.127-134, 1996.

LOPES, R.M.F.; FREITAS, V.L.O; LEMOS FILHO, J.P. Biometria de frutos e sementes e germinação de Plathymenia reticulata Benth. e Plathymenia foliolosa Benth. (Fabaceae - Mimosoideae).

Revista Árvore, v.34, n.5, p.797-805, 2010.

LORENZI, H. Árvores brasileiras: manual de identificação e cultivo de plantas arbóreas do Brasil. 5.ed. Nova Odessa: Instituto Plantarum, 2008. v.1., 385p.

MIRANDA, H.S.; SATO, M.N.; NETO, W.N.; AIRES, F.S. Fires in the Cerrado, the Brazilian savanna. In: COCHRANE, M.A. (Ed.). Tropical fire ecology: climate change, land use and ecosystem dynamics. Heidelberg: Springer-Praxis, 2009. p.427-450.

MUNHOZ, C.B.R.; AMARAL, A.G. Efeito do fogo no estrato herbáceo-subarbustivo do Cerrado In: MIRANDA, H.S. (Org.). Efeitos do regime do fogo sobre a estrutura de comunidades de cerrado: resultados do Projeto Fogo. Brasília: IBAMA, 2010. p.93-102.

OVERBECK, G.E.; MÜLLER, S.C.PILLAR, V.D.; PFADENHAUER, J. No heat-stimulated germination found in herbaceous species from burned subtropical grassland. Plant Ecology, v.184, n.2, p.237-243, 2006.
PALERMO, A.C. Efeitos do fogo na sobrevivência de sementes e na produção de frutos de Qualea parviflora Mart. (Vochysiaceae). 2011. 65f. Dissertação (Mestrado em Ecologia) - Universidade de Brasília, Brasília, 2011.

PIÑA-RODRIGUES, F.C.M.; FIGLIOLIA, M.B.; PEIXOTO, M.C. Teste de qualidade. In: FERREIRA, A.G.; BORGHETTI, F. (Ed.).

Germinação: do básico ao aplicado. Porto Alegre: Artmed, 2004. p.283-297.

POPINIGIS, F. Fisiologia da semente. 2.ed. Brasília: AGIPLAN, 1985.289p.

RAMOS, A.E. Efeito do fogo bienal e quadrienal na estrutura populacional e reprodução de quatro espécies vegetais do cerrado sensu stricto. 2004. 157f. Tese (Doutorado em Ecologia) Universidade de Brasília, Brasília, 2004.

RIBEIRO, M.L.; SALES, V.A.; MIRANDA, F.S.; SOARES, C.E.A.; OLIVEIRA, S.C.C. Influência da predação de sementes na germinação de leguminosas (Fabaceae) no Cerrado. Revista Brasileira de Biociências, v.5, n.S2, p.279-281, 2007.

SANTANA, D.G.; RANAL, M.A. Análise da germinação: um enfoque estatístico. Brasília: Universidade de Brasília, 2004. 248p.

SASSAKI, R.M.; FELIPPE, G.M. Single fruits and seedling establishment in Dalbergia miscolobium Benth. (Papilionaceae). Biotropica, v.31, n.4, p.591-597, 1999.

SATO, M.N.; MIRANDA, H.S.; MAIA, J.M. O fogo e o estrato arbóreo do Cerrado: efeitos imediatos e de longo prazo. In: MIRANDA, H.S. (Org.). Efeitos do regime do fogo sobre a estrutura de comunidades de cerrado: resultados do Projeto Fogo. Brasília: IBAMA, 2010. p.77-91.

SCHMIDT, I.B.; SAMPAIO, A.B.; BORGHETTI, F. Efeitos da época de queima sobre a reprodução sexuada e estrutura populacional de Heteropterys pteropetala (Adr. Juss.), Malpighiaceae, em áreas de cerrado sensu stricto submetidas a queimas bienais. Acta Botanica Brasilica, v.19, n.4, p.929-936, 2005. 
SILVA JÚNIOR, M.C. 100 Árvores do

Cerrado: guia de campo. Brasília: Rede de Sementes do Cerrado, 2005. 278p.
ZAIDAN, L.B.P.; CARREIRA, R.C. Seed germination in Cerrado species. Brazilian Journal of Plant Physiology, v.20, n.3, p.167-181, 2008. 